\title{
The Mitochondrial Antisense ncRNAs are Down-Regulated in Early Cervical Carcinoma
} \author{
Zapata $^{5}$ and Luis O. Burzio ${ }^{1,2,3 *}$ \\ ${ }^{1}$ Andes Biotechnologies SA, Zañartu 1482, Santiago, Chile \\ ${ }^{2}$ Fundación Ciencia para la Vida, Santiago, Chile \\ ${ }^{3}$ Facultad de Ciencias Biológicas, Universidad Andrés Bello, Santiago, Chile \\ ${ }^{4}$ Clinica Alemana, Santiago, Chile \\ ${ }^{5}$ Anatomo-pathology Unit, Hospital Barros Luco Trudeau, Santiago, Chile
}

Jaime Villegas ${ }^{1,2,3 *}$, Komal Dadlani ${ }^{1,2}$, Rodolfo Avila ${ }^{1,2}$, Claudio Villota ${ }^{1,2,3}$, Verónica Burzio ${ }^{1,2,3}$, Constanza Lopez ${ }^{1,2}$, Miguel Socias ${ }^{4}$, Luis

\begin{abstract}
Background: Cervical cancer is the third most common disease and the fourth leading cause of death in female's worldwide. Treatment of this disease is successful when detection is at early neoplasic stages. Hence, multiple efforts have been pursued for early detection of cervical neoplasia. The aim of this study was to analyze the differential expression of mitochondrial non-coding RNAs or ncRNA (sense and antisense) in normal and neoplastic cervical biopsies as a potential tool for diagnostic of cervical cancer.

Methods: The expression pattern of the sense and the antisense mitochondrial ncRNAs in cervical biopsies was carried out by chromogenic in situ hybridization (ISH). We examined 17 normal cervical tissues, 108 early and late neoplasias and 24 invasive cervical carcinomas. The hybridization results were compared with the diagnostic of each specimen carried out by pathologists.

Results: Like normal human keratinocytes, normal cervical epithelium expresses the sense and the antisense mitochondrial ncRNAs at the basal layer of the epithelium. Interestingly, ISH reveals that the antisense transcripts are always localized in the nucleus of basal cells in normal cervical epithelium. Early and late cervical intraepithelial neoplasia as well as invasive cervical carcinoma expresses the sense transcript. In contrast, the antisense transcripts are down-regulated in early and late neoplasia and in invasive cervical carcinoma.
\end{abstract}

Conclusions: This pilot study indicates that down-regulation of the antisense mitochondrial transcripts at early stages of cervical neoplasia can be explored as a diagnostic tool for early cervical neoplasia.

Keywords: Mitochondria; ncRNAs; Diagnosis; FISH; ISH; Human papilloma virus; Cervical cancer; Early cervical neoplasia

\section{Introduction}

Cervical cancer is the third most commonly diagnosed cancer and the fourth leading cause of cancer death in female's worldwide. In 2008, this disease accounts for 529,000 new cancer cases and 275,000 cancer deaths among females in 2008 [1]. About $85 \%$ of these cases and deaths occur in developing countries including South America, Eastern, Western, and Southern Africa, and South and Central Asia [1]. Epidemiological studies have shown that cervical infection with highrisk human papilloma viruses (HPV16 and HPV18) is necessary for the development of cervical cancer or its precursor cervical intraepithelial neoplasia or CIN [2,3]. HPV16 and HPV18 account for approximately $70 \%$ of all cervical cancers, while about ten other carcinogenic HPV account for the remaining 25\%-30\% [2-6]

Cytological screening or Papanicolaou test (Pap) has drastically reduced the incidence and mortality from cervical cancer. Since the introduction of the Pap test in the United States, cervical cancer incidence and mortality has been reduced markedly [7]. The Pap test together with histological studies of cervical biopsies has determined the different stages preceding cervical squamous carcinoma known as cervical intraepithelial neoplasia. These stages (CIN1, CIN2 and CIN3) correspond to the progressive invasion of the squamous cervical epithelium from the basal cell layer to the surface of the squamous epithelium. With the exception of HPV DNA determination by in situ hybridization [8] or liquid hybridization [9], there are no specific biomarkers to identify early stages of cervical neoplasia. However, recent efforts for new biomarkers have been reported, including the tumor suppressor $\mathrm{p} 16^{\mathrm{INK} 4 \mathrm{~A}}$, survivin and the minichromosome maintainance 5 or Mcm5 among other [10-14]. A very interesting finding reported recently is that the expression of the transcription factor E2 of HPV16 was detected in CIN1 and CIN2 but not in CIN3, suggesting a new diagnostic tool for early cervical neoplasia [15].

Human cells express a unique family of mitochondrial long ncRNAs (ncmtRNAs) [16,17]. Interestingly, these mitochondrial transcripts exit the organelle and are found in the cytoplasm and in the nucleus, suggesting a functional role of these molecules outside the organelle [18]. One of these transcripts, named sense ncmtRNA (SncmtRNA) is expressed in normal proliferating cells and tumor cells but not in resting cells, suggesting a functional role of this molecule in cell cycle progression $[16,17,19]$. Normal proliferating cells express,

*Corresponding authors: Jaime E. Villegas, Andes Biotechnologies SA Zañartu 1482, Santiago, Chile, Tel: 56-2-2367 2097; Fax: 56-2-237 2259; E-mail: jvillegas@bioschile.cl

Luis O. Burzio, Andes Biotechnologies SA, Santiago, Chile, E-mail: Iburzio@gmail. com

Received May 04, 2013; Accepted May 28, 2013; Published May 30, 2013

Citation: Villegas J, Dadlani K, Avila R, Villota C, Burzio V, et al. (2013) The Mitochondrial Antisense ncRNAs are Down-Regulated in Early Cervical Carcinoma J Cancer Sci Ther S7: 004. doi:10.4172/1948-5956.S7-004

Copyright: ( 2013 Villegas J, et al. This is an open-access article distributed under the terms of the Creative Commons Attribution License, which permits unrestricted use, distribution, and reproduction in any medium, provided the original author and source are credited. 
in addition to the SncmtRNA, two antisense mitochondrial ncRNAs (ASncmtRNA-1 and ASncmtRNA-2) [17]. Strikingly however, the ASncmtRNAs are down-regulated in human cancer cells regardless of cellular or tissue of origin [17]. Here we investigated the differential expression of the SncmtRNA and the ASncmtRNAs in biopsies of normal cervical epithelium, CIN1 to CIN3 and invasive cervical carcinoma (ICC).

\section{Methods}

\section{Tissue specimens}

Neutral-buffered formalin-fixed (10\%) and paraffin-embedded (FFPE) cervical specimens obtained from cervical punch biopsies, cervical conization and hysterectomy were selected from the archives of the Unit of Pathology of Hospital Barros Luco Trudeau. This study was approved by the Medical Ethical Committee of the same Hospital and the Ethical Committee of Fundación Ciencia y Vida. A total of 155 specimens were analyzed including 17 normal cervical tissues, 40 CIN1, 46 CIN2, 28 CIN3 and 24 cases of invasive cervical carcinoma (ICC). Diagnosis of sections $(5 \mathrm{~m})$ was carried out by two independent pathologists according to the classification criteria of the WHO.

\section{Cell culture}

Normal human keratinocytes (HFK), HeLa and SiHa cells were cultured according to ATCC guidelines. Cultures were maintained in a humidified incubator at $37^{\circ} \mathrm{C}$ and $5 \% \mathrm{CO} 2[17,19]$.

\section{Fluorescent in situ hybridization (FISH)}

Hybridization of HFK, HeLa and SiHa cells was carried out as described before $[17,19,20]$. Briefly, after trypsinization cells were recovered by centrifugation at $200 \times g$ for $10 \mathrm{~min}$ at room temperature (RT). The cell was resuspended in $100 \mu \mathrm{HCl} 0,2 \mathrm{~N}$ for $5 \mathrm{~min}$ at RT follow by neutralization with PBS. Cells were recovered by centrifugation and resuspended $100 \mu \mathrm{l}$ hybridization buffer (50\% formamide, $150 \mu \mathrm{g} / \mathrm{ml}$ herring sperm DNA, 4X SSC and 2 mM EDTA) containing $0,5 \mu \mathrm{M}$ 5'-Alexa fluor 488-labeled probe P1 (5' GTTCTTGGGTGGGTGTGGG $\left.3^{\prime}\right)$, complementary to the SncmtRNA and 0,05 $\mu \mathrm{M}$ each of two 5' Texas Red-labeled probes P2 (5'GATAACAGCGCAATCCTATT3') and P3 (5'ACCGTGCAAAGGTAGCATAATCA 3'), complementary to the ASncmtRNAs. Hybridization was performed for $15 \mathrm{~min}$ at $37^{\circ} \mathrm{C}$ (20). After washing in 2x SSC buffer, the cells were stained with DAPI (4',6-diamidino-2-phenylindole) and recovered by centrifugation. Samples were analyzed by fluorescence microscopy on an Olympus BX-51 microscope with a $63 x$ objective.

\section{Chromogenic in situ Hybridization.}

For tissue samples, $5 \mu \mathrm{m}$ thick serial paraffin sections were collected on silanized slides (DAKO) and deparaffinized in 2 consecutive 5 min xylene washes. One section was stained with hematoxylin and eosin (H\&E). The others were rehydrated in two 3-min washes of 98\% and 90\% ethanol each, and once in DEPC-treated distilled water for $5 \mathrm{~min}[16,17]$. Sections were then incubated in $2.5 \mu \mathrm{g} /$ $\mathrm{mL}$ of Proteinase K (Invitrogen) at RT for $20 \mathrm{~min}$ and then washed twice for $3 \mathrm{~min}$ in DEPC-treated water, immersed in $96 \%$ ethanol for $10 \mathrm{~s}$, and air dried [16,17]. Hybridization was carried out with digoxigenin-labeled probes 4 (5' TGATTATGCTACCTTTGCACGGT $\left.3^{\prime}\right)$ to detect the SncmtRNA and digoxigenin-labeled probes 5 (5' ACCGTGCAAAGGTAGCATAATCA 3') to detect the ASncmtRNAs. Hybridization mixtures contained 35 pmoles $/ \mathrm{mL}$ of probe 4 or probe 5 in hybridization solution ( $50 \%$ formamide, $150 \mu \mathrm{g} / \mathrm{ml}$ herring sperm DNA, 4X SSC, 1x Denhardt and 2 mM EDTA) was carried out overnight [17]. Washing and color development were carried out as described before [17].

\section{Results}

\section{Expression in normal cervical epithelium}

As reported before normal keratinocytes express the SncmtRNA and the ASncmtRNAs (Figure 1A). In contrast, FISH of HeLa and SiHa cells transformed with HPV18 and HPV16, respectively, reveals down-regulation of the ASncmtRNAs (Figure 1A). Then, we studied the expression pattern of these mitochondrial transcripts in normal cervical epithelium. Figure 1B shows the result of ISH of representative normal cervical epithelium. Notice that the hybridization signals are close to the basal layer of the epithelium and that the ASncmtRNAs hybridization signal is localized in the nucleus of both specimens (Figure 1B, arrows).

\section{Expression of the mitochondrial transcripts in CIN and ICC.}

Next, we investigated the expression of the SncmtRNA and the ASncmtRNAs in CIN1 and CIN2. The hybridization signal shows that as early as CIN1, the ASncmtRNAs are down-regulated as compare with normal cervical epithelium (Figure 2, CIN1). The expression of the SncmtRNAs is observed close to the basal layer of the epithelium. The same expression pattern was observed in CIN2. In these specimens, the hybridization signal corresponding to SncmtRNA is evident from the basal layer to the upper layer of the squamous cervical epithelium, while the hybridization signal of the ASncmtRNAs is negligible (Figure 2, CIN2) (Table 1).
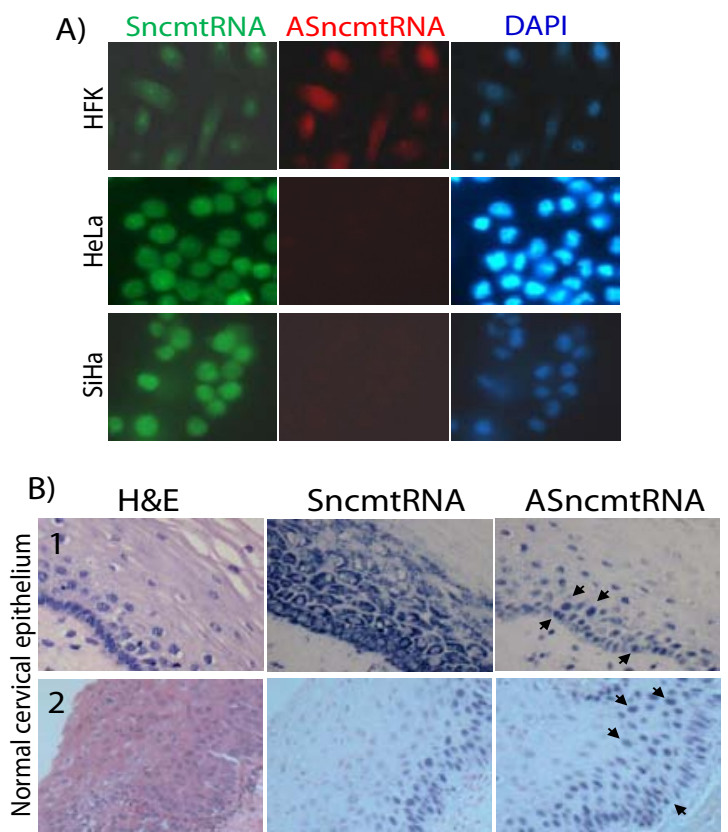

Figure 1: Expression of the mitochondrial transcripts in normal and tumor cells. A) HFK, HeLa and SiHa cells were subjected to FISH with probes specific for the SncmtRNA and the ASncmtRNAs. Only HFK expresses the ASncmtRNAs (red fluorescence) (63x). The cells were also staining with DAPI. B) ISH of normal cervical epithelium. The SncmtRNA and the ASncmtRNAs are expressed in basal layer of the epithelium compared with the section stained with H\&E. Notice that in both specimens the ASncmtRNAs are localized in the nucleus (arrows) (40x). 
In two specimens of CIN3, the hybridization signal of the SncmtRNA is evident from the basal layer to the upper layer of the squamous cervical epithelium (Figure $3 \mathrm{~A}$ ). The hybridization signal in specimen 1 reaches the outer layer of the squamous epithelium, while in specimen 2 the hybridization signal reaches the middle layer of the epithelium. On the other hand, the expression of the SncmtRNA in both representative specimens of ICC coincides with the tumor tissue as revealed by $\mathrm{H} \& \mathrm{E}$ staining (Figure $3 \mathrm{~B}$ ). No hybridization signal was observed in CIN3 and ICC with the probe targeted to the ASncmtRNAs (Figure $3 \mathrm{~A}$ and $3 \mathrm{~B}$ ).

\section{Discussion}

Although the Pap smear test has been extremely useful to reduce cervical cancer incidence, the test is prone to several errors resulting in high number of false-negative results. Therefore, there is an urgent need to investigate new specific biomarkers for early and late cervical neoplasia [21]. Several biomarkers have been studied to differentiate between early and late CIN including the tumor suppressor p16 $6^{\mathrm{INK} 4 \mathrm{~A}}$ $[10,11]$, the inhibitor of apoptosis survivin $[12,13]$ and Cdc6 and Mcm5 involve in DNA replication [14] among others. Another interesting study is the expression of HPV16 E2 protein using a specific polyclonal antibody. The expression of E2 seems to discriminate between early and late cervical neoplasia [15].

Here we reported that normal cervical squamous epitheliums express the SncmtRNA and the ASncmtRNAs, similar to the expression pattern of these transcripts in other normal human tissues [17]. In contrast, ISH indicate that the ASncmtRNAs are down-regulated as early as CIN1 and the same occurs in CIN2, CIN3 and ICC. However, it's important to mention that in approximately $15 \%$ of the specimens of CIN1, CIN2, CIN3 and ICC, the ISH signal of the SncmtRNA and the ASncmtRNAs was weak or absent. Some of these samples were subjected to ISH with specific probes for $18 \mathrm{~S}$ ribosomal RNA, a cellular transcript that should be present in normal and tumor cells. However these samples were also negative for the ribosomal RNA (data not
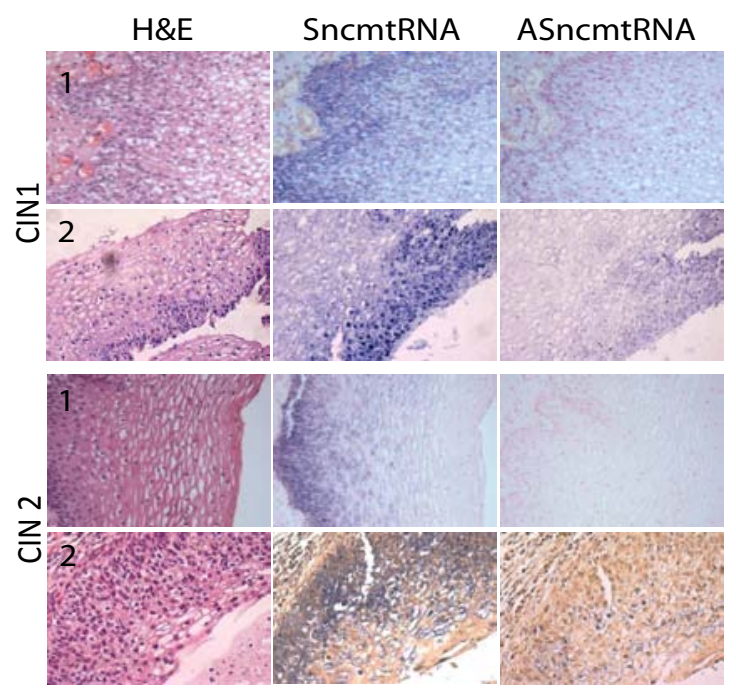

Figure 2: The ASncmtRNAs are down-regulated in CIN1 and CIN2. A) ISH of two representative specimens of CIN1 reveals the expression of the SncmtRNA in the basal layer of the epithelium and down-regulation of the ASncmtRNAs. B) The results show the expression of the mitochondrial transcripts in two specimens of CIN2. ISH reveals expression of the SncmtRNA and downregulation of the ASncmtRNAs (40x).

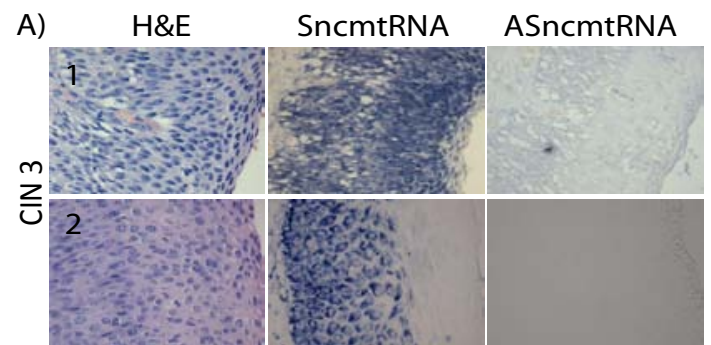

B)

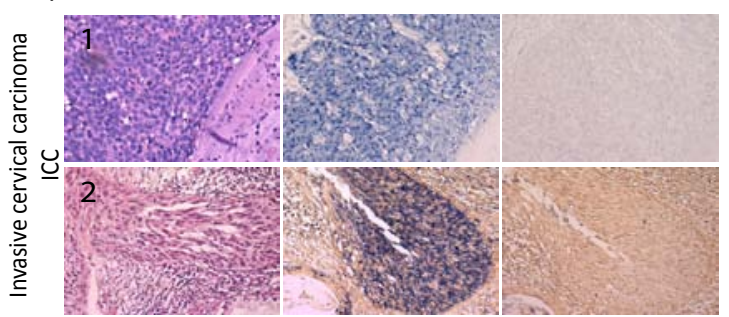

Figure 3: The ASncmtRNAs are down-regulated in CIN3 and ICC. A) ISH reveals expression of the SncmtRNA and down-regulation of the ASncmtRNAs in two specimens of CIN3. The hybridization signal of the SncmtRNA is on the basal and middle layer of the epithelium. B) The results show down-regulation of the ASncmtRNAs in two specimens of ICC. The hybridization signal of the SncmtRNA covers the complete tumor tissue as compare with H\&E staining (40x).

\begin{tabular}{|l|l|l|l|}
\hline \multicolumn{3}{|c|}{ Expression (\% Positive signal)* } \\
\hline & N cases & SncmtRNA & ASncmtRNAs \\
\hline Normal tissue & 17 & $15 / 17(+)(88 \%)$ & $15 / 17(+)(88 \%)$ \\
\hline CIN1 & 40 & $34 / 40(+)(85 \%)$ & $34 / 40(-)(85 \%)$ \\
\hline CIN2 & 46 & $41 / 46(+)(89 \%)$ & $41 / 46(-)(89 \%)$ \\
\hline CIN3 & 28 & $26 / 28(+)(92 \%)$ & $26 / 28(-)(92 \%)$ \\
\hline ICC & 24 & $19 / 24(+)(79 \%)$ & $19 / 24(-)(79 \%)$ \\
\hline
\end{tabular}

* In about $15 \%$ of the specimens, the hybridization signal was weak or absent (see text).

Table 1: ISH analyses of SncmtRNA and the ASncmtRNAs expression in norma cervical tissue, in CIN1, CIN2, CIN3 and in ICC

shown). Quality of the biopsy depends of multiple factor including type and time of fixation and the chemical quality of formalin, factors that are difficult to control.

Early down-regulation of the ASncmtRNAs in CIN1 is closely correlated with our previous studies in keratinocytes immortalized with the whole genome of HPV16 or HPV18 [19]. In these cells, the ASncmtRNAs are down-regulated similarly to their HPV-transformed counterparts, $\mathrm{SiHa}$ and HeLa cells [16]. Moreover, we showed that the oncoprotein E2 of HPV16 or HPV18 is involved in down-regulation of these transcripts [19]. These results are interesting in relations with the expression of E2 in early and late neoplasia of the cervix. The group of Thierry, using a specific polyclonal antibody against E2 of HPV16, has shown a close correlation between the expression of this viral protein and early and late CIN [15]. Thus, they show that E2 is not expressed in normal epithelium which is opposite to the expression of the ASncmtRNAs (Figure 1B). E2 is expressed in CIN1 and CIN2, which coincide with down-regulation of the ASncmtRNAs. However, although E2 is not expressed in CIN3 and ICC, the ASncmtRNAs remains down-regulated. Perhaps another cellular factor(s) in CIN3 
and ICC would have the ability to induce down-regulation of the ASncmtRNAs. Support to this hypothesis is the tumorigenic cell line TC-1, a mouse lung epithelial cell line immortalized with HPV-16 E6 and E7 and transformed with H-Ras oncogene [22]. The ASncmtRNAs are down-regulated in TC-1 tumor cells. Since transduction of HFK with E6 and E7 does not affect the expression of the ASncmtRNAs [19], down-regulation of these transcripts has to be mediated by $\mathrm{H}$-Ras.

Another interesting result in HPV-immortalized keratinocytes is the expression of new sense transcript named SncmtRNA-2. This transcript is expressed in HPV16- or HPV18-immortalized cells but not in HFK, SiHa, HeLa cells or other tumor cell lines [19]. Our previous results indicate that SncmtRNA-2 results from processing of SncmtRNA-1 outside mitochondria. In addition, we showed that the expression of the SncmtRNA-2 depends on the expression of both E6 and E7 of HPV [19]. Therefore, it may be interestingly to evaluate the expression of the SncmtRNA-2 in early and late cervical neoplasia as well as in cervical tumors. At present, we are planning a prospective study on the expression of the SncmtRNA-2 in cervical biopsies and cervical cytological smears. Taken together, the results presented here support the possibility to use the differential expression of these mitochondrial ncRNAs as a diagnostic tool for cervical cancer.

\section{Acknowledgements}

This work was supported by FONDEF Grants D10i1090 and D04i1338; FONDECYT Grants 11090060, 1110835; CCTE-PFB16 Program, CONICYT, Chile, and Grant DI-100-12/R, Universidad Andrés Bello, Chile.

\section{References}

1. Jemal A, Bray F, Center MM, Ferlay J, Ward E, et al. (2011) Global cancer statistics. CA Cancer J Clin 61: 69-90.

2. Walboomers JM, Jacobs MV, Manos MM, Bosch FX, Kummer JA, et al. (1999) Human papillomavirus is a necessary cause of invasive cervical cancer worldwide. J Pathol 189: 12-19.

3. de Sanjose S, Quint WG, Alemany L, Geraets DT, Klaustermeier JE, et al. (2010) Retrospective International Survey and HPV Time Trends Study Group. Human papillomavirus genotype attribution in invasive cervical cancer: a retrospective cross-sectional worldwide study. Lancet Oncol 11: 1048-1056.

4. Maucort-Boulch D, Franceschi S, Plummer M; IARC HPV Prevalence Surveys Study Group (2008) International correlation between human papillomavirus prevalence and cervical cancer incidence. Cancer Epidemiol Biomarkers Prev 17: 717-720.

5. Saslow D, Solomon D, Lawson HW, Killackey M, Kulasingam SL, et al. (2012) American Cancer Society, American Society for Colposcopy and Cervical Pathology, and American Society for Clinical Pathology Screening Guidelines for the Prevention and Early Detection of Cervical Cancer. CA Cancer J Clin 1-26.

6. Siegel R, Naishadham D, Jemal A (2012) Cancer statistics 2012. CA Cancer J Clin 62: 10-29.

7. Soumya D, Kumar A (2011) A Brief Assessment on Cervical Cancer. J Cancer Sci \& Ther S17.

8. Jacquemier J, Penault F, Durst M, Parc P, Seradour B et al. (1990) Detection of five different human papillomavirus types in cervical lesions by in situ hybridization. A study of 110 cases using sulfonated probes. Hum Pathol 21 : 911-917.
9. Schiffman MH, Kiviat NB, Burk RD, Shah KV, Daniel RW, et al. (1995) Accuracy and interlaboratory reliability of human papillomavirus DNA testing by hybrid capture. J Clin Microbiol 33: 545-550.

10. Tsoumpou I, Arbyn M, Kyrgiou M, Wentzensen N, Koliopoulos G, et al. (2009) p16(INK4a) immunostaining in cytological and histological specimens from the uterine cervix: a systematic review and meta-analysis. Cancer Treat Rev 35: 210-220.

11. Dijkstra MG, Heideman DA, de Roy SC, Rozendaal L, Berkhof J, et al. (2010) p16(INK4a) immunostaining as an alternative to histology review for reliable grading of cervical intraepithelial lesions. J Clin Pathol 63: 972-977.

12. Barbosa LC, da Silva ID, Corrêa JC, Ribalta JC (2011) Survivin and telomerase expression in the uterine cervix of women with human papillomavirus-induced lesions. Int J Gynecol Cancer 21: 15-21.

13. Branca M, Giorgi C, Santini D, Di Bonito L, Ciotti M, et al. (2005) Survivin as a marker of cervical intraepithelial neoplasia and high-risk human papillomavirus and a predictor of virus clearance and prognosis in cervical cancer. Am J Clin Pathol 124: 113-121.

14. Williams GH, Romanowski P, Morris L, Madine M, Mills AD, et al. (1998) Improved cervical smear assessment using antibodies against proteins that regulate DNA replication. Proc Natl Acad Sci U S A 95: 14932-14937.

15. Xue Y, Bellanger S, Zhang W, Lim D, Low J, et al. (2010) HPV16 E2 is an immediate early marker of viral infection, preceding E7 expression in precursor structures of cervical carcinoma. Cancer Res 70: 5316-5325.

16. Villegas J, Burzio V, Villota C, Landerer E, Martinez R, et al. (2007) Expression of a novel non-coding mitochondrial RNA in human proliferating cells. Nucleic Acids Res 35: 7336-7347.

17. Burzio VA, Villota C, Villegas J, Landerer E, Boccardo E, et al. (2009) Expression of a family of noncoding mitochondrial RNAs distinguishes normal from cancer cells. Proc Natl Acad Sci U S A 106: 9430-9434.

18. Landerer E, Villegas J, Burzio VA, Oliveira L, Villota C, et al. (2011) Nuclea localization of the mitochondrial ncRNAs in normal and cancer cells. Cell Oncol (Dordr) 34: 297-305

19. Villota C, Campos A, Vidaurre S, Oliveira-Cruz L, Boccardo E, et al. (2012) Expression of mitochondrial non-coding RNAs (ncRNAs) is modulated by high risk human papillomavirus (HPV) oncogenes. J Biol Chem 287: 21303-21315.

20. Rivas A, Burzio V, Landerer E, Borgna V, Gatica S, et al. (2012) Determination of the differential expression of mitochondrial long non-coding RNAs as a noninvasive diagnosis of bladder cancer. BMC Urol 12: 37

21. Frost M, Jarboe EA, Orlicky D, Gianani R, Thompson LC, et al. (2002) Immunohistochemical localization of survivin in benign cervical mucosa cervical dysplasia, and invasive squamous cell carcinoma. Am J Clin Patho 117: 738-744.

22. Lin KY, Guarnieri FG, Staveley-O'Carroll KF, Levitsky HI, August JT, et al. (1996) Treatment of established tumors with a novel vaccine that enhances major histocompatibility class II presentation of tumor antigen. Cancer Res 56 : $21-26$. 